\title{
2-DEOXY-D-GLUCOSE ENHANCES DICHLOROACETATE ANTITUMOR ACTION AGAINST LEWIS LUNG CARCINOMA
}

\author{
O.N. Pyaskovskaya*, D.L. Kolesnik, A.G. Fedorchuk, I.V. Prochorova, G.I. Solyanik \\ R.E. Kavetsky Institute of Experimental Pathology, Oncology and Radiobiology, NAS of Ukraine, \\ Kyiv 03022, Ukraine
}

\begin{abstract}
Aerobic glycolysis that supports high proliferation rate and survival of tumor cells in unfavorable conditions is among fundamental features of tumor metabolism. The search for active modulators of energetic metabolism capable of suppressing tumor growth and metastasis could result in higher effectiveness of anticancer therapy. Aim: To study antitumor and antimetastatic activity of the modulators of energetic metabolism dichloroacetate (DCA) and 2-deoxy-D-glucose (2DG) used in combination treatment of Lewis lung carcinoma (LLC). Materials and Methods: As experimental tumor model, LLC/R9 variant was used. DCA and 2DG were administered per os to $\mathrm{C} 57 \mathrm{Bl} / 6$ mice 5 times per week for 3 weeks at a total dose of 1.5 and $0.98 \mathrm{~g} / \mathrm{kg}$, respectively, as single agents or in combination starting from the following day after tumor cell transplantation. Growth of primary tumor and number and volume of lung metastases were registered. Lactate and pyruvate content was determined by enzymatic methods using lactate dehydrogenase. Electron paramagnetic resonance was used for analyzing the functional state of the components of mitochondrial respiratory chain. Engulfing activity and reactive oxygen species (ROS) production in tumor-associated $\mathrm{CD}^{+}{ }^{+}$cells was analyzed by flow cytometer with the use of FITC-labeled staphylococcus, and by spectrofluorometry with the use of 2.7 -dichlorofluorescein diacetate, respectively. Results: DCA administered as a single agent did not affect primary tumor growth but decreased the number and volume of lung metastases by $60 \%(\mathrm{p}<0.05)$ and $90 \%(\mathrm{p}<0.05)$, respectively. In mice treated with $2 \mathrm{DG}$ only, primary tumor volume as well as the number and volume of lung metastases were not affected. Combination treatment with DCA and 2DG resulted in the decrease of primary tumor volume, the number and volumes of lung metastases by $70 ; 46$, and $90 \%$, respectively $(p<0.05)$. High antitumor activity of DCA + 2DG was associated with $31 \%$ decrease $(p<0.05)$ of lactate content in tumor tissue and $120 \%$ increase $(\mathrm{p}<\mathbf{0 . 0 1})$ of ROS production in $\mathrm{CD}_{14}^{+}$cells recruited to the region of tumor growth. Conclusion: 2DG that possesses neither antitumor nor antimetastatic activity against LLC/R9 significantly enhanced antitumor activity of DCA with accompanying inhibition of glycolysis and increase of cytotoxic activity of $\mathrm{CD}^{+} 4^{+}$cells infiltrating tumor tissue. Taking into account significant antimetastatic activity of DCA this substance could be considered as a promising antimetastatic agent.
\end{abstract}

Key Words: Lewis lung carcinoma, antitumor and antimetastatic action, dichloroacetate, 2-deoxy-D-glucose, reactive oxygen species, $\mathrm{CD}_{14}^{+}$macrophage-like cells.

It is well known that glycolysis is the main way of ATP generation in the large majority of malignant cells even in the presence of oxygen. Despite the fact that special features of tumor bioenergetics are being used in oncological practice for diagnostic and prognostic purposes since $50^{\text {th }}$ of last century until now [1], only recently tumor metabolism has started to be considered as a therapeutic target [2]. As soon as glycolysis has been recognized as the main metabolic way providing tumor cell proliferation and survival in unfavorable conditions, the research has been focused on the compounds capable to suppress tumor growth via modification of its energetic metabolism. Among such compounds one could mention sodium dichloroacetate (DCA), an inhibitor of pyruvate dehydrogenase (PDH) kinase, and 2-deoxy-D-glucose (2DG), an inhibitor of glycolysis. Usually DCA has been used for correction of chronic metabolic malfunctions, but several data $[3,4]$ prompted to consider DCA as a promising anticancer agent capable of activating

Submitted: July 15, 2016.

*Correspondence: E-mail: pyaskovskaya@gmail.com Abbreviations used: 2DG - 2-deoxy-D-glucose; DCA - dichloroacetate; EPR - electron paramagnetic resonance; LDH - lactate dehydrogenase; LLC/R9 - high angiogenic variant of Lewis lung carcinoma; MtETC - mitochondrial electron transport chain; $\mathrm{PDH}$ - pyruvate dehydrogenase; PDK - pyruvate dehydrogenase kinase; ROS - reactive oxygen species. indirectly the enzymes of PDH complex and consequently shifting tumor cell metabolism from glycolysis to oxidative phosphorylation. 2DG is a structural analog of glucose in which hydroxyl group in the second position is replaced with hydrogen. Due to its structure, $2 D G$ is irreversibly phosphorylated with hexokinase into 2DG-phosphate that is accumulated in a cell without further transformation leading to block ATP production via glycolysis.

Up-to-date anticancer activity of DCA and 2DG has been demonstrated against many types of malignant cells in vitro and in vivo [3,5-7], and both compounds are being tested in clinical trials of phases I-II [8, 9]. However, so far, the use of these compounds as single anticancer agents did not show apparently positive results and their effectiveness in experimental tumors was insufficient [10-12]. Therefore is seems reasonable to study these compounds in combination with other antitumor agents to enhance their anticancer efficacy and to decrease their toxicity; such approach has shown promising results [13].

The aim of present study was to analyze antitumor and antimetastatic activity of DCA combined with 2DG against Lewis lung carcinoma (LLC).

\section{MATERIALS AND METHODS}

Experimental animals and tumor cells. The study was carried out on C57BI/6 mice 2-2.5 months 
old weighting $18.5-21.5 \mathrm{~g}$, bred at animal facility of R.E. Kavetsky Institute of Experimental Pathology, Oncology and Radiobiology (IEPOR, Kyiv, Ukraine). The use and care of the experimental animals have been performed in accordance with the standard international rules of biologic ethics and was approved by Institutional Animal Care and Use Committee.

As experimental tumor model, we have used LLC variant LLC/R9, resistant to cisplatin and generated from the parental LLC strain by multiple sequential cycles of cisplatin administration to tumor-bearing mice in vivo [14]. LLC/R9 cells were maintained in vitro in RPMI 1640 culture medium (Sigma, USA) supplemented with $10 \%$ fetal bovine serum (FBS) (Sigma, USA) and $40 \mu \mathrm{g} / \mathrm{ml}$ gentamycin at $37^{\circ} \mathrm{C}$ in humidified atmosphere with $5 \% \mathrm{CO}_{2}$.

For in vivo experiments, LLC/R9 cells were propagated in vitro at standard conditions and inoculated i.m. to the mice $\left(10^{6}\right.$ cells in $0.1 \mathrm{ml}$ of Hank's solution per animal).

The number of cells in suspension and their viability were routinely evaluated using hemocytometer and $0.4 \%$ trypan blue solution.

Agents, dosages and administration schedule. DCA (Sigma, USA) and 2DG (Sigma, USA) were administered per os at total doses of 1.5 and $0.98 \mathrm{~g} / \mathrm{kg}$, respectively (both doses corresponded to therapeutical range and were well tolerated [15-17]) at the regimens of a single use or combined treatment.

After tumor transplantation, experimental animals were randomly distributed in four groups: group 1 mice treated with DCA $(n=11)$, group $2-$ mice treated with 2DG $(n=13)$, group 3 - mice treated with DCA in combination with $2 D G(n=13)$. In the last case, $2 D G$ was administered at least $3 \mathrm{~h}$ after DCA. Mice treated with water at the same regimen and in the same volume served as the control $(n=12)$.

Treatment with DCA and 2DG has been initiated on the following day after tumor cell transplantation, 5 times per week for 3 weeks. DCA and 2DG solutions were prepared ex tempore in water, and were administered in a volume of $0.4 \mathrm{ml} / \mathrm{animal}$.

Antitumor and antimetastatic activity of the studied compounds were evaluated on day 21 after tumor transplantation. Primary tumor diameter was measured triply per week starting from the moment of development of palpable tumors. Tumor volume (V) was calculated by the formula:

$$
V=0.52 d^{3} \text {, }
$$

where $d$ - tumor diameter.

Anticancer activity was evaluated as tumor growth inhibition rate calculated by the formula:

$$
\left[\left(V_{k}-V\right) / V_{k}\right] \cdot 100 \% \text {, }
$$

where $V, V_{k}$ - average tumor volumes in experimental and control groups, respectively.

The number and volume of lung metastases was routinely analyzed using binocular microscope and millimeter scale.

Volume of metastases (V) was calculated by the formula:
$V=\sum_{T}^{N} \pi d_{i}^{3} /\left[n_{i} \times 6\right]$,

where $n_{i}-$ number of metastases with the diameter of $d_{i}$.

Functional state of the components of mitochondrial respiratory chain of tumor cells was analyzed using electron paramagnetic resonance (EPR). Tumor tissue was cut into the samples of cylindrical shape $(\mathrm{d}=4.0 \mathrm{~mm}, \mathrm{l}=25-35 \mathrm{~mm})$, frozen and stored at $-70^{\circ} \mathrm{C}$. EPR analysis of the samples was performed at $77 \mathrm{~K}$ using spectrometer E-109 Varian (USA) at potential sweep speed of $500 \mathrm{E} / \mathrm{min}$, modulation amplitude of $1.25 \cdot 10 \mathrm{E}$, power of super high frequency radiation of $10.0 \mathrm{~mW}$, constant session of apparatus of $1.0 \mathrm{~s}$. The levels of reduced nonheme iron-sulfur (Fe-S) centers ( $\mathrm{g}=1.94$ ) of MtETC proteins, nitrosyl (NO) complexes of heme iron $\left(g_{\text {med }}=2.007\right)$ and nonheme iron $\left(g_{\text {med }}=2.03\right)$ were determined by the data of EPR spectra.

Contents of glucose, creatinine and urea in blood plasma samples were determined with the use of semiautomatic biochemical analyzer. Blood plasma was prepared using heparinized tubes and stored at $-20^{\circ} \mathrm{C}$ until the time of analysis.

Glucose content in tumor tissue homogenates was determined by enzyme glucose-oxidant method using the kit for glucose analysis in biologic fluids (Sigma, USA) according to instructions of the manufacturer.

Lactate and pyruvate content in tumor tissue homogenates was determined by enzyme spectrophotometry method using lactate dehydrogenase (Sigma, USA) [18].

The samples for biochemical study were collected from 4-5 animals per group, frozen and stored at $-20^{\circ} \mathrm{C}$ (blood plasma) or in liquid nitrogen (tumor tissue) until the moment of analysis.

Total activity of lactate dehydrogenase (LDH) in tumor tissue homogenates was determined by the rate of NADH (Sigma, USA) oxidation registered by spectrophotometry by the decrease of optical density at the wavelength of $340 \mathrm{~nm}$ [18].

Engulfing capacity of tumor-associated CD $14^{+}$ cells was determined by flow cytometry with the use of FITC-labeled staphylococcus.

Tumor-associated $C D 14^{+}$cells were identified with the use of anti-CD14 antibodies (Becton Dickinson, USA) according to the instructions of the manufacturer [19].

Production of reactive oxygen species (ROS) intumor-associated $\mathrm{CD} 14^{+}$cellswas determined byspectrofluorometry with the use of 2.7-dichlorofluorescein diacetate (Sigma, USA) [19].

Statistical analysis of the data was performed by descriptive statistics, Student's $t$-test and Mann Whitney $U$ test, with the use of Microsoft Excel and Microcal Origin programs.

\section{RESULTS AND DISCUSSION}

It has been shown that combination of DCA and 2DG exerted significant antitumor activity, while both 
studied compounds as single agents were practically inactive in inhibiting primary tumor growth. The data on the influence of DCA, 2DG and their combination on the primary tumor growth and metastasis at $21^{\text {st }}$ day after LLC transplantation are presented in Fig. 1. As one may see, at this time in LLC/R9-bearing mice treated with DCA or 2DG the primary tumor volume did not differ significantly from that in control group (see Fig. 1). Meanwhile, DCA combined with 2DG significantly inhibited tumor growth starting from the $16^{\text {th }}$ day and on the $21^{\text {st }}$ day tumor volume was by $70 \%$ $(p<0.05)$ lower compared to the control.

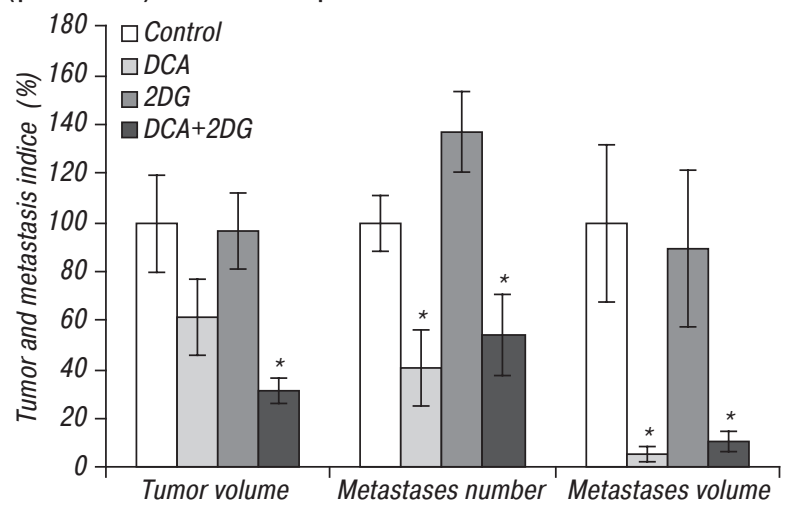

Fig. 1. Effect of DCA, $2 D G$ and their combination on tumor growth and metastasis indices in LLC/R9 bearing mice. ${ }^{*} p<0.05$ as compared to the control

In regard to antimetastatic effects, only DCA but not 2DG suppressed metastasizing. In animals treated with DCA the number and volume of lung metastases decreased nearly by $60 \%(p<0.05)$ and more than by $90 \%$ ( $p<0.05$ ), respectively, as compared to the control indexes. Meanwhile, no antimetastatic activity was revealed in 2DG treated mice (see Fig. 1). In the case of combined treatment of animals with 2DG and DCA antimetastatic effects were related to activity of DCA only, but not 2DG: in this group of mice the number and volume of lung metastases decreased by $46 \%(p<0.05)$ and nearly by $90 \%(p<0.05)$, respectively, compared to the control group.

The study of lactate and pyruvate levels in the tumor tissue has shown that antitumor activity of DCA combined with 2DG was mediated by their significant effect on glucose metabolism reflected in decreased lactate level and accumulation of pyruvate in the tumor. As shown in Fig. 2, combined treatment of mice with DCA and 2DG resulted in $31 \%$ decrease $(p<0.05)$ of lactate content and $48 \%$ increase $(p<0.05)$ of pyruvate content compared to the control values. Such effects could reflect the decreased utilization of pyruvate in the tricarboxylic acid cycle and/or decreased LDH activity in the reactions of pyruvate to lactate reduction. As far as LDH activity did not change significantly upon combined use of DCA and 2DG (Fig. 2), accumulation of pyruvate could be related to the decreased intensity of the reactions initiated by pyruvate as a substrate. This could be accompanied not only with pyruvate accumulation, but also with an increased pool of NAD-H and the decreased pool of NAD+ which is an electron acceptor providing glycolysis function- ing. It couldn't be excluded that altered level of lactate and pyruvate in tumor tissue may be related to the competition between 2DG and glucose.

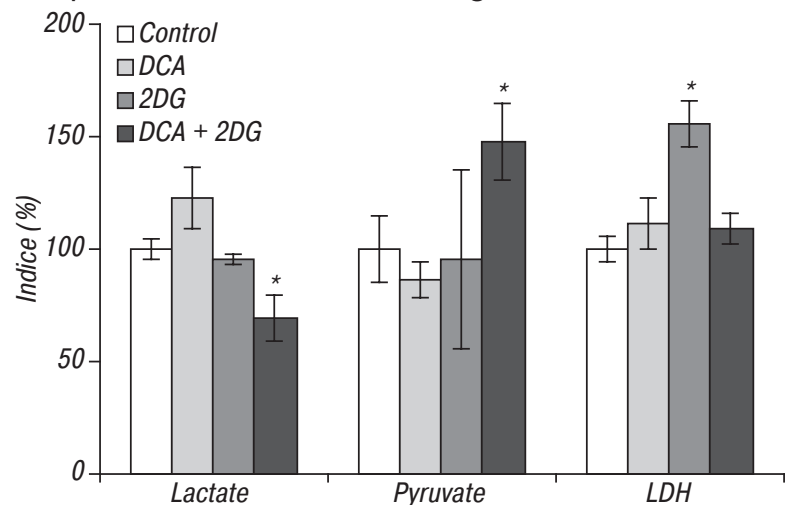

Fig. 2. Effect of DCA, $2 D G$ and their combination on lactate and pyruvate content and LDH activity in tumor tissue of LLC/ R9 bearing mice. ${ }^{*} \mathrm{p}<0.05$ as compared to the control

In the group of animals treated with DCA and 2DG, lactate content decreased not only in tumor tissue but also in blood plasma (by 38\% compared to the control, $p<0.05$, Table 1). The decrease of lactate content in blood plasma close to $30 \%(p<0.05)$ was also observed in the group of mice treated with 2DG only indicating the contribution of 2DG into the decrease of lactate content in the tumor and blood plasma of animals treated by 2DG and DCA combination.

Table 1. Effect of DCA, 2DG and their combination on blood plasma biochemical indices of LLC/R9 bearing mice

\begin{tabular}{lcccc}
\hline Group of mice & Glucose, mM & Lactate, $\mathrm{mM}$ & $\begin{array}{c}\text { Creatinine, } \\
\mathrm{mM}\end{array}$ & Urea, mM \\
\hline Control & $5.3 \pm 0.9$ & $8.5 \pm 0.9$ & $31.8 \pm 1.5$ & $4.5 \pm 0.5$ \\
DCA & $5.7 \pm 0.8$ & $8.2 \pm 0.5$ & $38.3 \pm 2.0^{\star}$ & $3.6 \pm 0.4$ \\
2DG & $6.5 \pm 0.3$ & $6.2 \pm 0.5^{\star}$ & $35.4 \pm 1.0$ & $4.2 \pm 0.5$ \\
DCA + 2DG & $4.8 \pm 1.1$ & $5.2 \pm 0.7^{\star}$ & $33.3 \pm 4.5$ & $3.1 \pm 0.6$ \\
\hline
\end{tabular}

Note: ${ }^{*} \mathrm{p}<0.05$ as compared to the control.

As shown in Table 1, decreased lactate content in blood plasma of mice treated with 2DG or 2DG combined with DCA, did not affect glucose content in blood plasma. At the same time, in animals treated with DCA we have registered significant increase of creatinine content $(p<0.05)$ suggesting an ability of this compound to cause kidney malfunction in tumor-bearing animals.

Earlier we have shown that high antitumor efficacy of DCA, alone or in combination with antiangiogenic aconitin-containing agent, against solid variant of Ehrlich carcinoma was related to the increased production of ROS in $\mathrm{CD}_{14}{ }^{+}$cells infiltrating the tumor with increased nitrosylation of proteins of mitochondrial ETC in tumor cells [20]. However, the studied agents have no effect on the functional state of mitochondrial ETC in LLC cells and nitrosylation levels of proteins of mitochondrial ETC. As one may see in Fig. 3 , in LLC/ R9 bearing animals treated with DCA combined with 2DG or DCA only, an intensity of EPR signals corresponding to NO-complexes of heme $\left(g_{\text {med }}=2.007\right)$ or nonheme $\left(g_{m e d}=2.03\right)$ iron in proteins of mitochondrial ETC were not statistically higher than analogous indexes in the control. Similar pattern has been observed for EPR signal intensity of Fe-S cluster proteins 
( $g=1.94)$, which, as it is known, characterize functional activity of ETC complex I: in all groups of animals this index was practically equal to that in the control.

Significant increase $(p<0.05)$ of nitrosylation level of heme and nonheme proteins has been registered only in the group of animals treated with 2DG, that may be attributed to a capability of this compound to modify the production of reactive nitrogen species [21]. However, an enhancement of antitumor activity of DCA with 2DG was not related to its influence on mitochondrial ETC functioning in LLC cells.

An analysis of a functional state of tumor-infiltrating $\mathrm{CD} 14^{+}$-macrophage like cells has shown that antitumor effect of DCA in combination with 2DG was realized, at least in part, via enhancement of their cytotoxic activity. In the group of animals treated with DCA in combination with 2DG, the level of ROS production in these cells increased by $120 \%(p<0.01)$ compared with that in the control (Fig. 4). In these groups of animals, the relative number of tumor-infiltrating $\mathrm{CD} 14^{+}$cells and their engulfing capacity did not differ significantly from corresponding indexes in the control. It is necessary to note that in the groups of mice treated with DCA or 2DG the number and functional activity of $\mathrm{CD} 14^{+}$ cells did not differ significantly from control group.

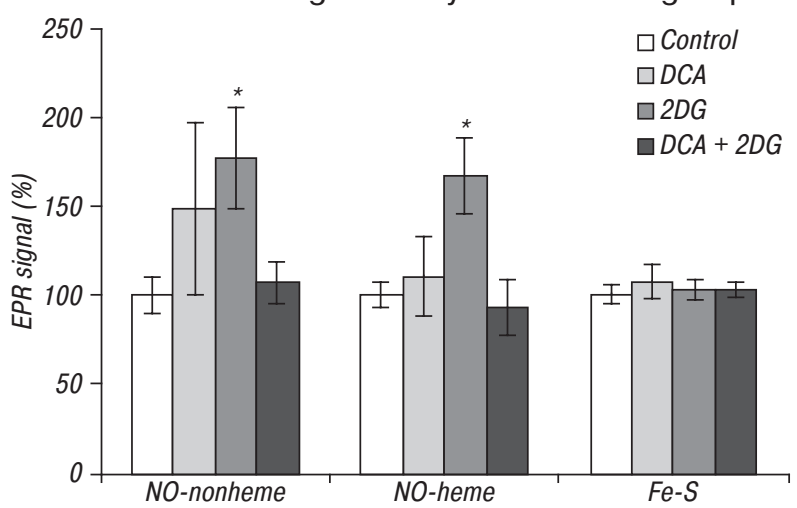

Fig. 3. Effect of DCA, 2DG and their combination on the indices of functional state of MtETC in the tumor cells. ${ }^{*} p<0.05$ as compared to the control

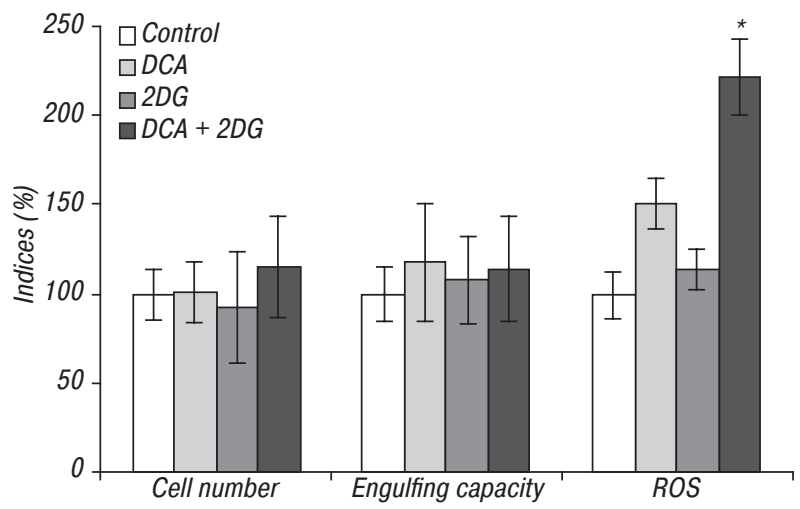

Fig. 4. Effect of DCA, $2 D G$ and their combination on the indices of functional state of tumor-infiltrating $\mathrm{CD}_{14}{ }^{+}$macrophage like cells. ${ }^{*} p<0.05$ as compared to the control

Therefore, the present work has demonstrated significant enhancement of antitumor action of DCA used in combination with other inhibitor of energetic metabolism, 2DG, even in the absence of antitumor effects of these agents used at the same dosages in monotherapy mode. According to literature data, antitumor activity of DCA is largely based on its capability for negative regulation of PDK, a negative regulator of enzymes of mitochondrial PDH complex, which plays a key role in regulation of tricarboxylic acid cycle and oxidative phosphorylation [22]. In the case of PDK inhibition with DCA, tumor cell metabolism switches from glycolysis to oxidative phosphorylation that should affect lactate production in tumor tissue. In our work, antitumor effectiveness of DCA in combination with 2DG was associated with inhibition of glycolysis, as it has been illustrated by the drop of lactate content in the tumor. However, the relation between antitumor activity of DCA and 2DG and their influence on tumor metabolism has been found only in the case of their combined use, supposedly due to the enhancement of pharmacological effects of these compounds. In other words, an effective inhibition of glycolysis in tumor cells sufficient for realization of antitumor action has been exerted as a consequence of simultaneous intensification of oxidative phosphorylation in tumor cells via DCA-induced activation of PDH complex and competitive inhibition of glucose consumption by tumor cells by 2DG, and consequently, decreased energy and/or concentration of glycolytic intermediates.

Antitumor effectiveness of DCA combined with 2DG could be related not only to glycolysis inhibition, but also to their effect on tumor microenvironment, namely, activation of tumor-infiltrating cells expressing CD14 receptor [23]. Capability of DCA to affect different types of immunocompetent cells, in particular, to cause alternative activation of tumor-associated macrophages has been shown in several studies [24, 25]. Our data evidenced on the capability of the studied agents to increase cytotoxic activity of tumor infiltrating CD14 ${ }^{+}$immunocompetent cells (reflected in increased intracellular production of ROS in these cells), while there was no influence of phagocytic (engulfing) activity of the cells. Such effect on the functional state of $\mathrm{CD} 14^{+}$cells was observed only in the case of the combined use of DCA and 2DG.

At last, one should note that while DCA-based monotherapy was found to be insufficient for achievement of antitumor effect, DCA, but not 2DG, exerted an expressed antimetastatic activity against LLC/ R9. Combined use of DCA and 2DG did not enhance such antimetastatic effect. Antimetastatic activity of DCA has been demonstrated in other studies, in particular, against rat mammary adenocarcinoma cells if administered by intravenous route [26]. Possible mechanism of antimetastatic activity of DCA was discussed in the study [27], where it has been shown that activation of mitochondrial PDH complex in tumor cells significantly increased their sensitivity to anoikis and, correspondingly, decreased their metastatic potential. Taking into account high antimetastatic activity of DCA against LLC/R9, capability of this agent to promote activation of PDH complex through PDK inhibition in tumor cells, and the data on the close relation between activity of PDH complex in tumor cells 
and their metastatic potential, one could suppose that DCA could be considered as a promising agent with high antimetastatic activity.

\section{REFERENCES}

1. Kim BS, Sung SH. Usefulness of 18 F-FDG uptake with clinicopathologic and immunohistochemical prognostic factors in breast cancer. Ann Nucl Med 2012; 26: 175-83.

2. Galluzzi L, Kepp O, Vander Heiden MG, Kroemer G. Metabolic targets for cancer therapy. Nat Rev Drug Discov 2013; 12: 829-46.

3. Michelakis ED, Webster L, Mackey JR. Dichloroacetate (DCA) as a potential metabolic-targeting therapy for cancer. Br J Cancer 2008; 99: 989-94.

4. Michelakis ED, Sutendra G, Dromparis P, et al. Metabolic modulation of glioblastoma with dichloroacetate. Sci Transl Med 2010; 2: 31-4.

5. Zhang XD, Deslandes E, Villedieu M, et al. Effect of 2-deoxy-D-glucose on various malignant cell lines in vitro. Anticancer Res 2006; 26: 3561-6.

6. Madhok BM. Dichloroacetate induces apoptosis and cellcycle arrest in colorectal cancer cells. BJC 2010; 102: 1746-52.

7. Saed GM, Fletcher NM, Jiang ZL, et al. Dichloroacetate induces apoptosis of epithelial ovarian cancer cells through a mechanism involving modulation of oxidative stress. Reprod Sci 2011; 18: 1253-61.

8. Raez LE, Papadopoulos K, Ricart AD, et al. A phase I dose-escalation trial of 2-deoxy-D-glucose alone or combined with docetaxel in patients with advanced solid tumors. Cancer Chemother Pharmacol 2013; 71: 523-30.

9. Chu QS, Sangha R, Spratlin J, et al. A phase I openlabeled, single-arm, dose-escalation, study of dichloroacetate (DCA) in patients with advanced solid tumors. Invest New Drugs 2015; 33: 603-10.

10. Cheong J-H, Park ES, Liang J, et al. Dual inhibition of tumor energy pathway by 2-deoxy glucose and metformin is effective against a broad spectrum of preclinical cancer models. Mol Cancer Ther 2011; 10: 2350-62.

11. Terse PS, Joshi PS, Bordelon NR, et al. 2-Deoxy-dglucose (2-DG)-induced cardiac toxicity in rat: NT-proBNP and BNP as potential early cardiac safety biomarkers. Int J Toxicol 2016; 35: 284-93.

12. Fedorchuk AG, Pyaskovskaya ON, Gorbik GV, et al. Effectiveness of sodium dichloroacetate against glioma C6 depends on administration schedule and dosage. Exp Oncol 2016; 38: 80-83.

13. Dai Y, Xiong X, Huang G, et al. Dichloroacetate enhances adriamycin-induced hepatoma cell toxicity in vitro and in vivo by increasing reactive oxygen species levels. PLoS ONE 2014; 9: e92962. doi:10.1371/journal.pone.0092962.
14. Pyaskovskaya ON, Dasyukevich OI, Kolesnik DL, et al. Changes in VEGF level and tumor growth characteristics during Lewis lung carcinoma progression towards cis-DDP resistance. Exp Oncol 2007; 29: 197-202.

15. Kumar K, Wigfield S, Gee HE, et al. Dichloroacetate reverses the hypoxic adaptation to bevacizumab and enhances its antitumor effects in mouse xenografts. J Mol Med (Berl) 2013; 91: 749-58.

16. Robey IF, Martin NK. Bicarbonate and dichloroacetate: Evaluating $\mathrm{pH}$ altering therapies in a mouse model for metastatic breast cancer. BMC Cancer 2011; 11: 235-44.

17. Vijayaraghavan R, Kumar D, Dube SN, et al. Acute toxicity and cardio-respiratory effects of 2-deoxy-D-glucose: a promising radio sensitiser. Biomed Environ Sci 2006; 19: $96-103$.

18. Biochemical methods (lipid and energy metabolism). In: Prohorova MI, ed. L: Leningrad Univ, 1982. 272 p. (in Russian).

19. Haitova RM. The use of flow cytometry to assess the functional activity of human immune system. Manual for physicians. Institute of Immunology 2001; 53 p. (in Russian).

20. Pyaskovskaya ON, Boychuk IV, Fedorchuk AG, et al. Modification of dichloroacetate antitumor activity against Ehrlich carcinoma with the use of aconitine-containing agent. Exp Oncol 2015; 37: 192-6.

21. Kurtoglu M, Gao N, Shang J, et al. Under normoxia, 2-deoxy-D-glucose elicits cell death in select tumor types not by inhibition of glycolysis but by interfering with $\mathrm{N}$-linked glycosylation. Mol Cancer Ther 2007; 6: 3049-58.

22. Stacpoole PW. The pharmacology of dichloroacetate. Metabolism 1989; 38: 1124-44.

23. Ziegler-Heitbrock HWL, Ulevitch RJ. CD14: cell surface receptor and differentiation marker. Immunol Today 1993; 14: 121-5.

24. Ohashi T, Akazawa T, Aoki M et al. Dichloroacetate improves immune dysfunction caused by tumor-secreted lactic acid and increases antitumor immunoreactivity. Int J Cancer 2013; 133: 1107-18.

25. Kumar A, Kant S, Singh SM. Antitumor and chemosensitizing action of dichloroacetate implicates modulation of tumor microenvironment: a role of reorganized glucose metabolism, cell survival regulation and macrophage differentiation. Toxicol Appl Pharmacol 2013; 273: 196-208.

26. Sun RC, Fadia M, Dahlstrom JE, et al. Reversal of the glycolytic phenotype by dichloroacetate inhibits metastatic breast cancer cell growth in vitro and in vivo. Breast Cancer Res Treat 2010; 120: 253-60.

27. Kamarajugadda S, Stemboroski L, Cai Q, et al. Glucose oxidation modulates anoikis and tumor metastasis. Mol Cell Biol 2012; 32: 1893-907. 